\title{
Design Methodology for Throughput Optimum Architectures of Hash Algorithms of the MD4-class
}

\author{
Yong Ki Lee ${ }^{(1)}$, Herwin Chan ${ }^{(1)}$, and Ingrid Verbauwhede ${ }^{(1),(2)}$ \\ (1) University of California, Los Angeles, USA \\ (2) Katholieke Universiteit Leuven, Belgium \\ \{jfirst, herwin, ingrid\}@ee.ucla.edu
}

\begin{abstract}
In this paper we propose an architecture design methodology to optimize the throughput of MD4-based hash algorithms. The proposed methodology includes an iteration bound analysis of hash algorithms, which is the theoretical delay limit, and DFG (Data Flow Graph) transformations to achieve the iteration bound. We applied the methodology to some MD4-based hash algorithms such as SHA1, MD5 and RIPEMD-160. Since SHA1 is the algorithm which requires all the techniques we show, we also synthesized the transformed SHA1 algorithm in a 0.18 $\mu m$ CMOS technology in order to verify its correctness and its achievement of high throughput. To the best of our knowledge, the proposed SHA1 architecture is the first to achieve the theoretical throughput optimum beating all previously published results. Though we demonstrate a limited number of examples, this design methodology can be applied to any other MD4-based hash algorithm.
\end{abstract}

\section{Introduction}

Hash algorithms not only have cryptographic key features such as the one way function property and collision resistance but also have operational key features such as high speeds and fixed output sizes independent of the input sizes. Due to these key features, hash algorithms are one of the most popular primitive components in the cryptographic systems. Especially they are commonly used in Digital Signature Algorithms [1] and message authentications. Designing a given hash algorithm to be throughput optimum is a critical issue. Considering that data sizes and communication speeds are increasing every year, a low throughput of hash algorithms can be a bottle neck in the digital and/or communications systems. Even though many architectures have been proposed, the analysis of theoretical limits has not 
been presented before the initiation by our previous work $[17,18]$. This lack of theoretical analysis may lead designers to make futile attempts to improve their architecture when it is already optimal.

In this paper, we propose a systematic design methodology for throughput optimum architectures for MD4-based hash algorithms, which include most commonly used hash algorithms such as SHA1, MD5 and RIPEMD-160. The proposed design methodology is composed of three steps. The first step is to construct a DFG (Data Flow Graph) of a given algorithm. In this process, we select a DFG which has the minimum iteration bound. The second step is to analyze the iteration bound, which defines the minimum delay in which an algorithm may run independent of implementation architectures. The last step is to construct an architecture achieving the iteration bound by transformations to the DFG. The retiming transformation and the unfolding transformation are used. The concepts of the iteration bound analysis of DFG and the transformations are publicly known techniques and detailed explanation can be found in [2], and we adopt most of the notations and the definitions from this reference. In this paper, however, we develop and formalize the concepts to specifically design MD4-based hash algorithms.

Moreover, we apply the design methodology to the popular MD4-based hash algorithms, SHA1, MD5 and RIPEMD-160, in order to thoroughly investigate the methodology usage. According to [3], "customized hash functions are those which are specifically designed "from scratch" for the explicit purpose of hashing, with optimized performance in mind, and without being constrained to reusing existing system components such as block ciphers or modular arithmetic. Those having received the greatest attention in practice are based on the MD4 hash function." In this paper we focus on this class of MD4 type hash functions. More specifically we use SHA1, MD5 and RIPEMD-160 as examples as they are most used in practice.

According to our analysis and design, optimal architectures for MD5 and RIPEMD-160 require only the retiming transformation while SHA1 requires both the retiming transformation and the unfolding transformation. Due to this property of SHA1, we synthesized the proposed architecture of SHA1 to verify the achievement of high throughput and the correctness of the transformations, which is done by checking the hash outputs. According to the best of our knowledge, the proposed architecture is the first throughput optimum architecture of SHA1. The result of synthesis shows a throughput of 3,738 Mbps, the highest throughput among all previously published papers.

The remainder of the paper is organized as follows. In Section 2, we review related works for high throughput architectures of hash algorithms and in Section 3, we present our design methodology for 
throughput optimum architectures of MD4-based hash algorithms. The proposed design methodology is applied to SHA1 in Section 4 and is applied to MD5 and RIPEMD-160 in Section 5. We show the synthesis results of the transformed SHA1 to verify our proposal in Section 6 and we conclude this paper in Section 7.

\section{Related Works}

There are several techniques to increase throughputs in MD4-based hash algorithms implementations. The most commonly used techniques are pipelining, loop unrolling and using Carry Save Adders (CSA): Pipelining techniques reduce critical path delays by properly positioning registers, whose applications can be found in [4-6]; Unrolling techniques improve throughputs by performing several iterations in single cycle [7-10]; CSA techniques reduce arithmetic addition delays of two or more consecutive additions [47,10]. Many of the published papers combine multiple techniques to achieve a higher throughput. SHA1 is implemented in [7, 10-14], SHA2 is implemented in $[4,5,8-10,13]$, MD5 is implemented in [12-16] and RIPEMD-160 is implemented in $[12,13,15]$.

Despite numerous proposals for high throughput hash implementations, a delay bound analysis had been neglected except for our pervious work $[17,18]$ and the architecture designs are mostly done by intuition. In [4], the authors present a design that achieves the iteration bound, though they do not claim optimality. In fact, their design is the last revision of several other suboptimal attempts [5]. In one of our previous work [17], the analysis of the iteration bound of SHA1 is done and in order to approach the iteration bound, the unfolding transformation is applied. However, it does not achieve the actual iteration bound and it required too much hardware duplication. This is caused by neglecting the retiming transformation and concentrating only on increasing the unfolding factor. In addition, the

CSA technique is not considered in the proposed architecture. In another our previous work [18], the iteration bound analysis of SHA2 is performed and its throughput optimum architecture is presented. In this work, due to the property of SHA2, the unfolding transformation was not necessary to achieve its iteration bound.

In this paper, we propose a systematic design methodology for MD4-based hash algorithms starting from a DFG (Data Flow Graph) and analyzing the iteration bound to design the optimum architecture. Since SHA1 requires both the retiming transformation and the unfolding transformation, applying the 
design methodology to SHA1 is a good example for a general MD-based hash algorithm. The synthesis result of SHA1 confirms the validity of the design methodology.

\section{Design Methodology for Throughput Optimization}

Our proposed design methodology adopts the iteration bound analysis and the transformations of DFG (Data Flow Graph), whose detailed concept and explanation can be found in [2]. We develop and formalize the technique to fit MD4-based hash algorithms. MD4-based hash algorithms are iterative algorithms. This means that the output of one iteration is the input for the next. Iterations in general restrict the usage of pipelining or other straightforward techniques to improve the throughput. In order to analyze the iteration bound, we represent a given algorithm with a DFG. To understand the methodology, we explain the design steps using a simple example.

\subsection{DFG Representation}

Eq. 1 shows the mathematical expression of our example. $A, B$ and $C$ are variables whose values are stored in registers and their indices represent the algorithm's iteration. $K$ is a fixed constant independent of the iteration. The next values of variables are updated using the current values of variables and the constant according to the operational equation. This type of equations is very common in MD4-based hash algorithms.

$$
\begin{aligned}
& A(n+1)=A(n)+B(n) *(C(n)+K) \\
& B(n+1)=A(n) \\
& C(n+1)=B(n)
\end{aligned}
$$

Fig. 1 shows the DFG of Eq. 1. The dashed lines are for driving outputs at the $n$-th iteration and the solid lines indicate the data flow throughout the DFG. The meaning of the bold lines will be explained in the next sub-section. Box $A, B$ and $C$ represent registers, which give the output at cycle $n$, and circles represent some functional nodes which perform the given functional operations. A $D$ on edges represents an algorithmic delay, i.e. a delay that cannot be removed from the system. Next to algorithmic delays, nodes also have functional delays. We express the functional delays, i.e. the delays to perform 


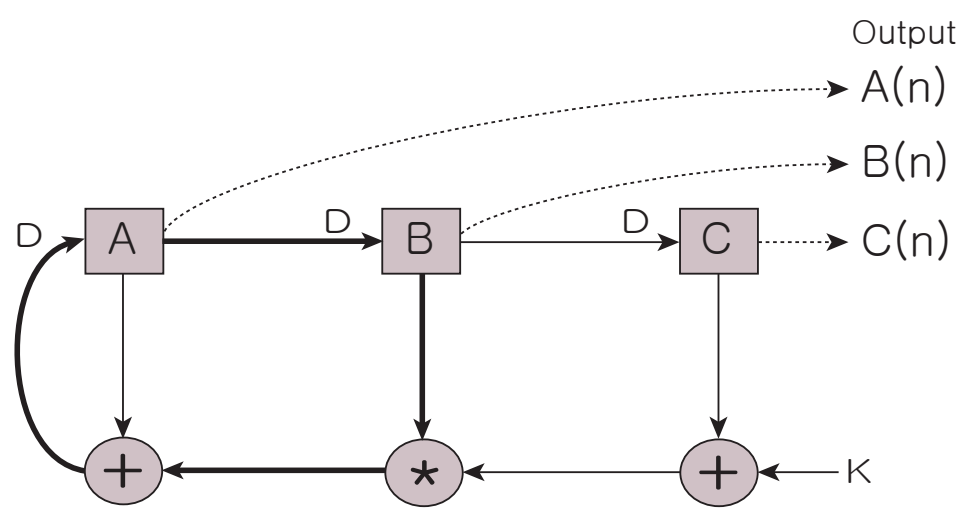

Fig. 1. An example of DFG

the given operations, of + and $*$ as $\operatorname{Prop}(+)$ and $\operatorname{Prop}(*)$ respectively. The binary operators, + and *, can be arbitrary operators but we assume $\operatorname{Prop}(+)<\operatorname{Prop}(*)$ in this example. The iteration bound analysis starts with an assumption that any functional operation is atomic. This means that a functional operation can not be split or merged into some other functional operations.

\subsection{The Iteration Bound Analysis}

A loop is defined as a path that begins and ends at the same node. In the DFG in Fig. 1, $\longrightarrow \longrightarrow+D A$, $A \longrightarrow{ }_{D} B \longrightarrow \longrightarrow+\underset{D}{\longrightarrow} A$ and $A \underset{D}{\longrightarrow} B \underset{D}{\longrightarrow} \longrightarrow+\longrightarrow * \longrightarrow+\underset{D}{\longrightarrow} A$ are the loops. The loop calculation time is defined as the sum of the functional delays in a loop. If $t_{l}$ is the loop calculation time and $w_{l}$ is the number of algorithmic delays in the $l$-th loop, the $l$-th loop bound is defined as $t_{l} / w_{l}$. The iteration bound is defined as follows:

$$
T_{\infty}=\max _{l \in L}\left\{\frac{t_{l}}{w_{l}}\right\}
$$

where $L$ is the set of all possible loops. The iteration bound creates a link between the arithmetic delay and the functional delay. It is the theoretical limit of a DFG's delay bound. Therefore, it defines the maximally attainable throughput. Please note that every loop needs to have at least one algorithmic delay in the loop otherwise the system is not causal and cannot be executed. 
Since the loop marked with bold line has the maximum loop delay assuming that $\operatorname{Prop}(+)<\operatorname{Prop}(*)$, the iteration bound is as follows:

$$
\begin{aligned}
T_{\infty} & =\max \left\{\operatorname{Prop}(+), \frac{\operatorname{Prop}(+)+\operatorname{Prop}(*)}{2}, \frac{2 \times \operatorname{Prop}(+)+\operatorname{Prop}(*)}{3}\right\} \\
& =\frac{\operatorname{Prop}(+)+\operatorname{Prop}(*)}{2}
\end{aligned}
$$

This means that a critical path delay in this DFG can not be less than this iteration bound. The critical path delay is defined as the maximum calculation delay between any two consecutive algorithmic delays, i.e. D's. In our example (Fig. 1), the critical path delay is $2 \times \operatorname{Prop}(+)+\operatorname{Prop}(*)$, which is larger than the iteration bound. The maximum clock frequency (and thus throughput) is determined by the critical path (the slowest path). The iteration bound is a theoretical lower bound on the critical path delay of an algorithm. We use the retiming and unfolding transformations to reach this lower bound.

\subsection{The Retiming Transformation}

The minimum critical path delay that can be possibly achieved using the retiming transformation is shown in Eq. 4.

$$
\left\lceil T_{\infty}\right\rceil=\left\lceil\frac{\operatorname{Prop}(+)+\operatorname{Prop}(*)}{2}\right\rceil=\operatorname{Prop}(*)
$$

Assuming that a functional node can not be split into multiple parts, $\lceil\cdot\rceil$ is the maximum part when $\operatorname{Prop}(+)+\operatorname{Prop}(*)$ is evenly distributed into $N$ parts, where $N$ is the number of algorithmic delays in a loop. This is denoted by the 2 in our example and sits in the denominator. Since the total delay $\operatorname{Prop}(+)+\operatorname{Prop}(*)$ can be partitioned into one delay $\operatorname{Prop}(+)$ and the other delay $\operatorname{Prop}(*)$, the attainable delay bound by the retiming transformation is $\operatorname{Prop}(*)$.

The retiming transformation modifies a DFG by moving algorithmic delays, i.e. D's, through the functional nodes in the graph. Delays of out-going edges can be replaced with delays from in-coming edges and vice versa. Fig. 2 shows the retiming transformation steps performed on Fig. 1. Based on the $B$ node in Fig. 1, the delay of the out-going edge is replaced with delays of the in-coming edges resulting in Fig. 2(a). By performing the retiming transformations based on the $C$ node and the right + node in Fig. 2(a), we obtain the DFG of Fig. 2(b). Another retiming transformation based on the $*$ node gives 
Fig. 2(c). Note that the out-going edges and the in-coming edges must be dealt as a set, and the edge connected by $K$ is excluded from the retiming transformation since $K$ is a fixed constant independent of $n$. In Fig. 2(a) the index of $B$ in output changed to $n+1$ since $B$ provides the output in advance of one cycle due to the retiming transformation. The index change of $C$ in Fig. 2(b) is also caused by the

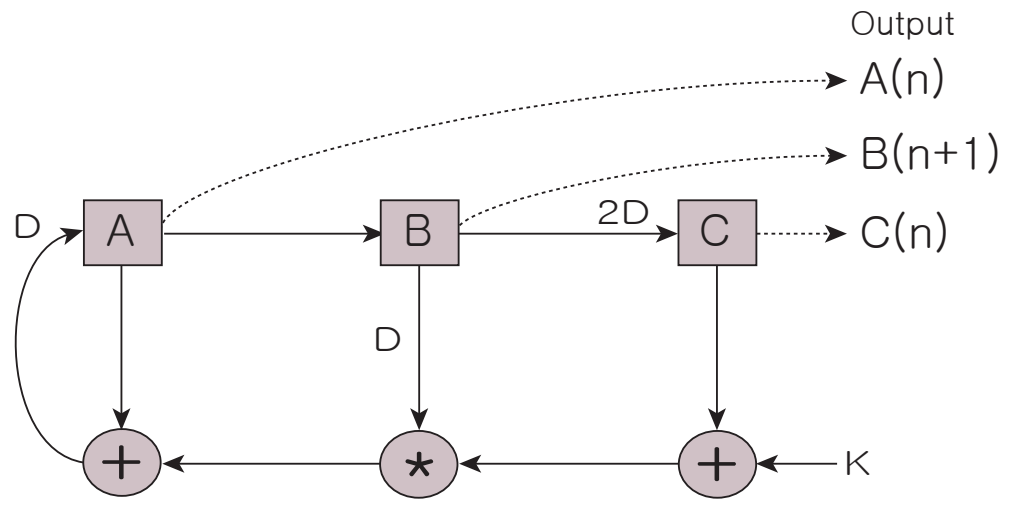

(a) Retimed based on $B$

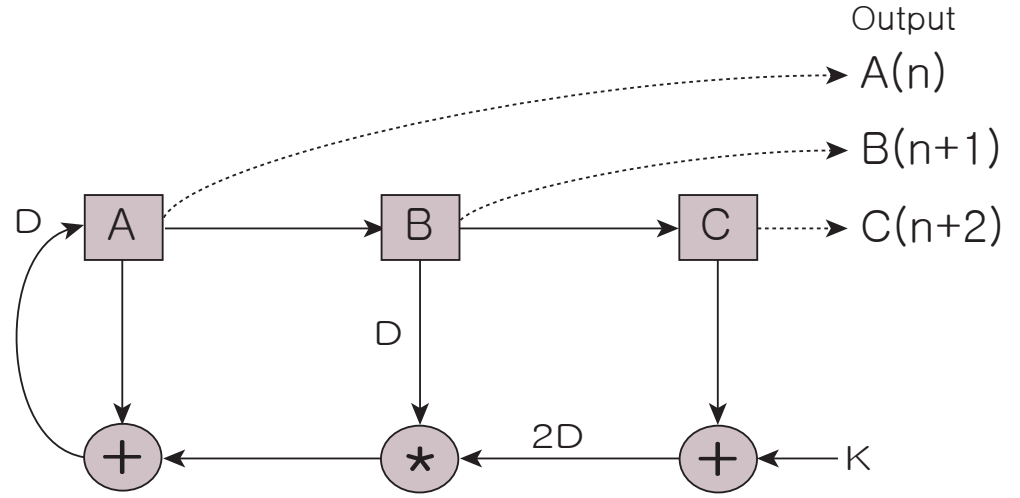

(b) Retimed based on $C$ and +

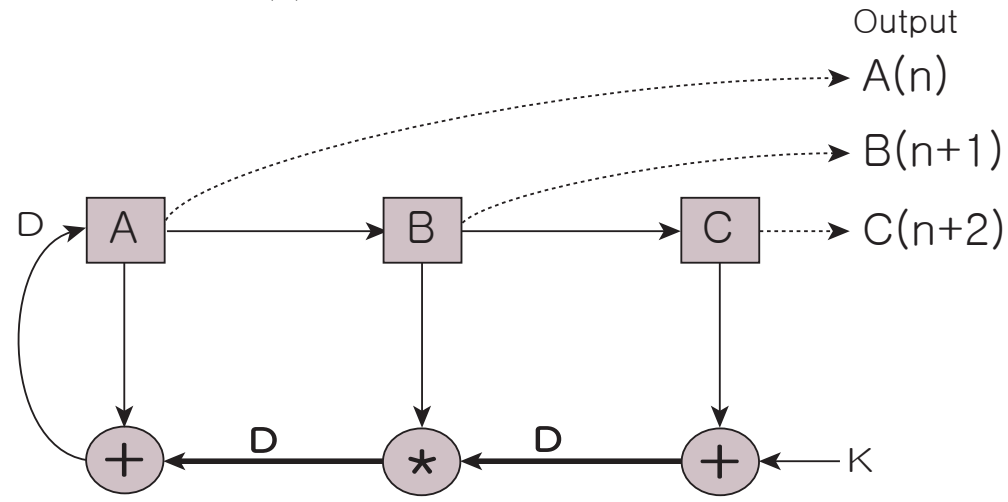

(c) Retimed based on *

Fig. 2. Retiming Transformation 
retiming transformation. Therefore, the critical path becomes the path between the two bolded $D$ 's in Fig. 2(c) and its delay is reduced to $\operatorname{Prop}(*)$, which is the same as Eq. 4. However, the iteration bound still has not been met.

\subsection{The Unfolding Transformation}

The unfolding transformation improves performance by calculating several iterations in a single cycle. The minimally required unfolding factor is the denominator of the iteration bound. This fact can be inferred by noting that the difference between Eq. 3 and Eq. 4 is caused by the un-cancelled denominator of the iteration bound. In our example, the required unfolding factor is two.

We expand Eq. 1 with the unfolding transformation by representing two iterations at a time, resulting in Eq. 5. While optimal architectures can be obtained by applying the unfolding transformation on the retimed DFG (Fig. 2), doing so involves complex reconstruction of the operational equation. Therefore, we decided to perform unfolding before retiming, which allows us to obtain optimality through a simpler path. The unfolding transformation applied on the original operational equation Eq. 1 produces Eq. 5.

$$
\begin{aligned}
\begin{aligned}
A(n+2) & =A(n+1)+B(n+1) *(C(n+1)+K) \\
& =A(n)+B(n) *(C(n)+K)+A(n) *(B(n)+K)
\end{aligned} \\
B(n+2)=A(n+1)=A(n)+B(n) *(C(n)+K) \\
C(n+2)=B(n+1)=A(n)
\end{aligned}
$$

Note that now $A(n+2), B(n+2)$ and $C(n+2)$ are expressed as a function of $A(n), B(n)$ and $C(n)$. By introducing a temporary variable Tmp, Eq. 5 can be simplified into Eq. 6.

$$
\begin{aligned}
& \operatorname{Tmp}(n)=A(n)+B(n) *(C(n)+K) \\
& A(n+2)=\operatorname{Tmp}(n)+A(n) *(B(n)+K) \\
& B(n+2)=\operatorname{Tmp}(n) \\
& C(n+2)=A(n)
\end{aligned}
$$




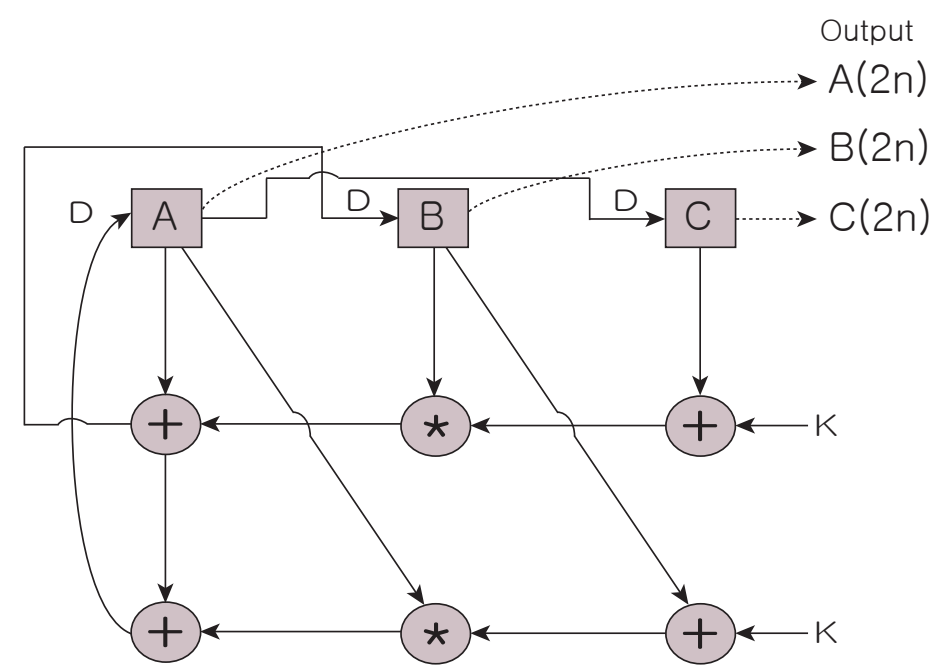

(a) Unfolding Transformation

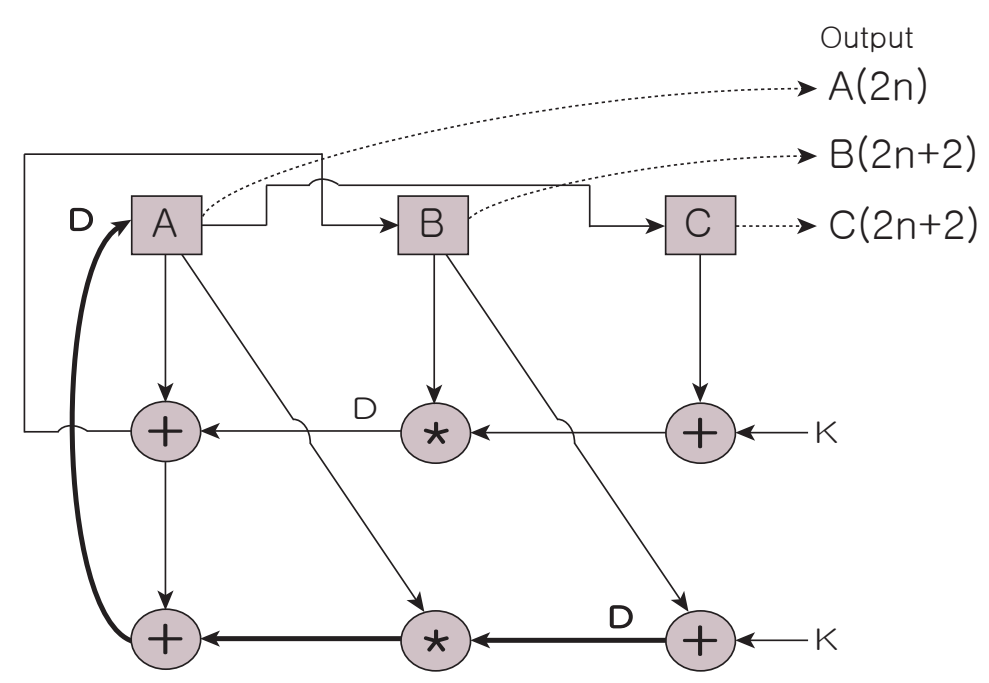

(b) Retiming Transformation

Fig. 3. Unfolding and Retiming Transformation

By doubling the number of functional nodes, we are able to unfold the DFG by a factor of two (Fig. 3(a)). Box $A, B$ and $C$ now give the outputs of every second iteration. By applying the retiming transformation to the unfolded DFG, the resulting critical path becomes the path in bold between the two bolded $D$ 's, which is $D \longrightarrow * \longrightarrow+\longrightarrow D$ (Fig. 3(b)). Therefore, the critical path delay is $\operatorname{Prop}(+)+\operatorname{Prop}(*)$. Due to the unfolding factor of two, the normalized critical path delay, $\hat{T}$, can be calculated by dividing the critical path delay by two as shown in Eq. 7. This final transformation results 
in an architecture that achieves the iteration bound of the example DFG (Fig. 1).

$$
\hat{T}=\frac{\operatorname{Prop}(+)+\operatorname{Prop}(*)}{2}=T_{\infty}
$$

Now the only remaining step is the implementation of the resulting DFG. Note that some of the square nodes are no longer paired with an algorithmic delay and that the output indexes are changed, which can be seen in Fig. 3(b). The explanation about how these issues is dealt with during implementation will be given in Section 6, where we synthesize the SHA1 algorithm.

\subsection{Assumption and Restriction of the Design Methodology}

The assumption and restrictions of our design methodology is directly inherited from the properties of the iteration bound analysis of DFG's. The analysis and architecture designs are limited up to the functional operations of a given hash algorithm. For example, in most MD4-based hash algorithms, additions and non-linear functions are used in iteration. The iteration bound analysis is agnostic about the specific implementations of functional operation such as the addition and non-linear functions; the absolute functional delay is not considered and the actual iteration delays will vary depending on the implementations of the functional operations. However, note that relative delay amounts between functional operations must be considered. As mentioned before, the reason is that we want to distribute the total functional delay as evenly as possible. Moreover, we assume that any two functional operations can not be merged or split to some other functional operations. If we want to use some other functional operation instead of given operations, e.g. using a CSA and an addition instead of two consecutive additions, then a new DFG must be derived and analyzed.

\section{Iteration Bound Analysis and Transformations of the SHA1 Algorithm}

In this section, we analyze the iteration bound of SHA1 and present the DFG which achieves the iteration bound.

\subsection{The SHA1 Algorithm}

SHA1 [20], which is one of the most popular hash algorithms, was issued by the National Institute of Standards and Technology (NIST) in 1995. SHA1 takes input data of length less than $2^{64}$ bits and gives 


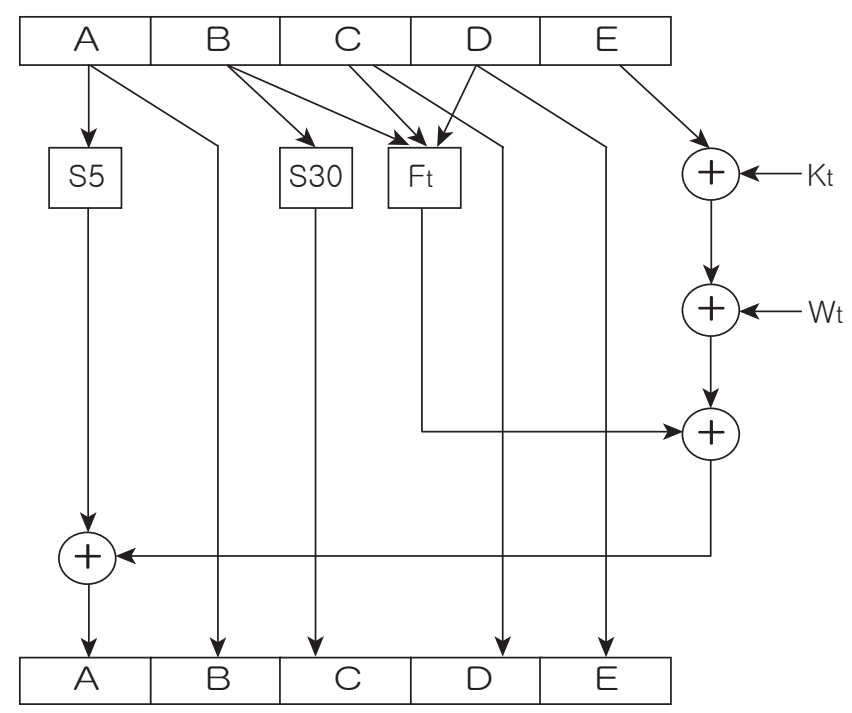

Fig. 4. SHA1 hash operation architecture

output of 160 bit length. After packing and padding an arbitrary length message into one or multiple 512 bit blocks, each block is processed separately. Each block requires 4 rounds of hash operations, and each round is composed of 20 hash operations. The differences among the rounds are on a scrambling constant, $K_{t}$, and a nonlinear operation, $F_{t}$, where $t$ represent the $t$-th hash operation. The architecture of one step hash operation is illustrated in Figure 4 and the mathematical expression is described in Eq. 8 .

$$
\begin{aligned}
& T E M P_{t}=S 5\left(A_{t}\right)+F_{t}\left(B_{t}, C_{t}, D_{t}\right)+E_{t}+W_{t}+K_{t} ; \\
& E_{t+1}=D_{t} ; \quad D_{t+1}=C_{t} ; \quad C_{t+1}=S 30\left(B_{t}\right) ; \quad B_{t+1}=A_{t} ; \quad A_{t+1}=T E M P_{t} ;
\end{aligned}
$$

In Fig. 4 and Eq. 8, $S 5$ and $S 30$ represent 5 and 30 circular left shifts respectively and $W_{t}$ is a 32 bit register value. The number of $W_{t}$ registers can be either 80 or 16 . If 80 registers are to be used, the $W_{t}$ values can be calculated before the hash operations, and if 16 registers are to be used, the $W_{t}$ values are dynamically updated during the hash operations. In this paper, all the syntheses are done using 16 registers. 


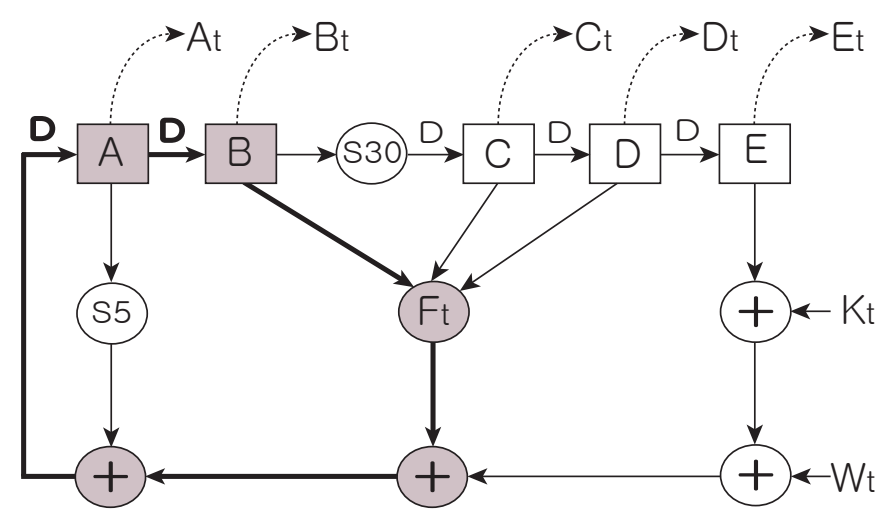

(a) DFG without CSA

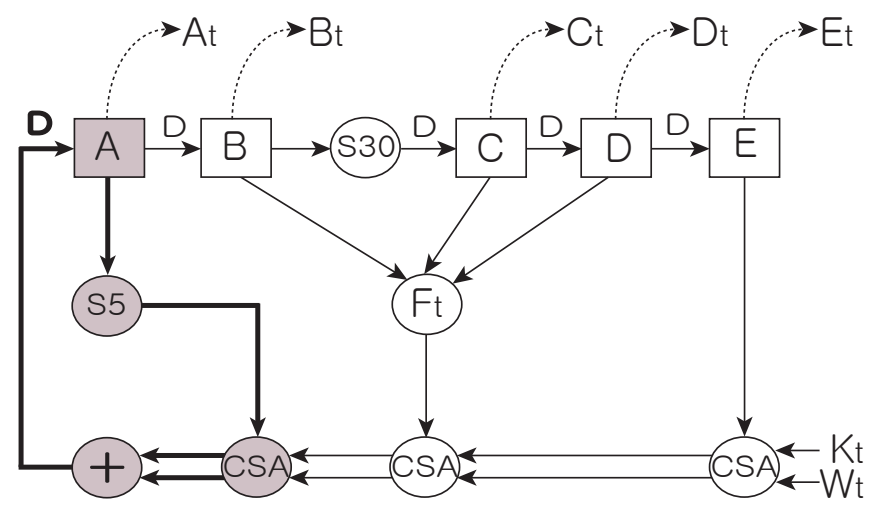

(b) DFG with CSA

Fig. 5. SHA1 Data Flow Graph

\subsection{The DFG and Iteration Bound of SHA1}

The data flow graph (DFG) representations of SHA1 are shown in Fig. 5. The square nodes $A, B, C$, $D$ and $E$ correspond to the register variables of the SHA1 algorithm. The circular nodes represent the individual functions in a hash operation. Dotted arrow lines are to drive outputs at time index $t$. Since the order of the four adders in SHA1 does not make a difference on the mathematical calculation, there are several different ways to represent a SHA1 DFG. For example, $(a+b)+c$ and $(b+c)+a$ will have different DFG's though they are mathematically equivalent. First of all a DFG of the minimum iteration bound must be chosen, then transformations are applied to achieve this bound. The DFG with the smallest iteration bound is shown in Fig. 5

Note that in iteration bound analysis, we assume that each functional node in a DFG can not be merged or split into some other operations. Therefore, in order to use a CSA (Carry Save Adder), we have 
to draw another DFG. A CSA produces two values (carry and sum) from three input operands. Since CSA has small area and propagation delay in throughput optimized implementations, it is commonly used when three or more operands are summed. In Fig. 5, (a) and (b) represent DFG's of each case, where the shaded nodes represent the maximum loop bound in each DFG.

We assume that $\operatorname{Prop}\left(F_{t}\right) \approx \operatorname{Prop}(C S A)<\operatorname{Prop}(+)$, which is reasonable since the worst case of $F_{t}$ is the "Three Input Bitwise Exclusive OR" operation and this is the same as the critical path of CSA, and a 32 bit addition has definitely larger critical path than the others. The iteration bounds of the two DFG's are as the following Eq. 9, where super-script (a) and (b) represent each DFG of Fig. 5. Since shifts are negligible in hardware implementations, we ignore the delays of shifts.

$$
\begin{aligned}
& T_{\infty}^{(a)}=\frac{2 \times \operatorname{Prop}(+)+\operatorname{Prop}\left(F_{t}\right)}{2}=\operatorname{Prop}(+)+\frac{\operatorname{Prop}\left(F_{t}\right)}{2} \\
& T_{\infty}^{(b)}=\operatorname{Prop}(+)+\operatorname{Prop}(C S A) \approx \operatorname{Prop}(+)+\operatorname{Prop}\left(F_{t}\right)
\end{aligned}
$$

Since $T_{\infty}^{(a)}<T_{\infty}^{(b)}$, the use of CSA alone does not help to reduce the iteration bound in this case.

\subsection{Applying the Retiming Transformation}

The attainable minimum critical path delay with the retiming transformation (not using the unfolding transformation) is as the following Eq. 10.

$$
\begin{aligned}
& \left\lceil T_{\infty}^{(a)}\right\rceil=\left\lceil\frac{2 \times \operatorname{Prop}(+)+\operatorname{Prop}\left(F_{t}\right)}{2}\right\rceil=\operatorname{Prop}(+)+\operatorname{Prop}\left(F_{t}\right) \\
& \left\lceil T_{\infty}^{(b)}\right\rceil=\operatorname{Prop}(+)+\operatorname{Prop}(C S A) \approx \operatorname{Prop}(+)+\operatorname{Prop}\left(F_{t}\right)
\end{aligned}
$$

Therefore, even though applying the CSA technique increases the iteration bound, the critical path delays of two cases are similar if there is no unfolding transformation. The DFG's which achieve these critical delays are shown in Fig. 6 . The time indices of $F, K$ and $W$ are changed due to the retiming transformation. The shaded nodes represent the critical path of each case, which are $A \longrightarrow B \longrightarrow F_{t-1} \longrightarrow+$ and $A \longrightarrow S 5 \longrightarrow C S A \longrightarrow+$. Note that there is no propagation delay on $A, B$ and $S 5$.

Though the two critical paths in Fig. 6 are similar, in practice (b) is preferred since CSA has smaller area than a throughput optimized adder. Note that the logic gates of CSA of 1 bit word are equivalent to those of a full adder which are the minimum gates for one bit addition. If an adder is implemented as 


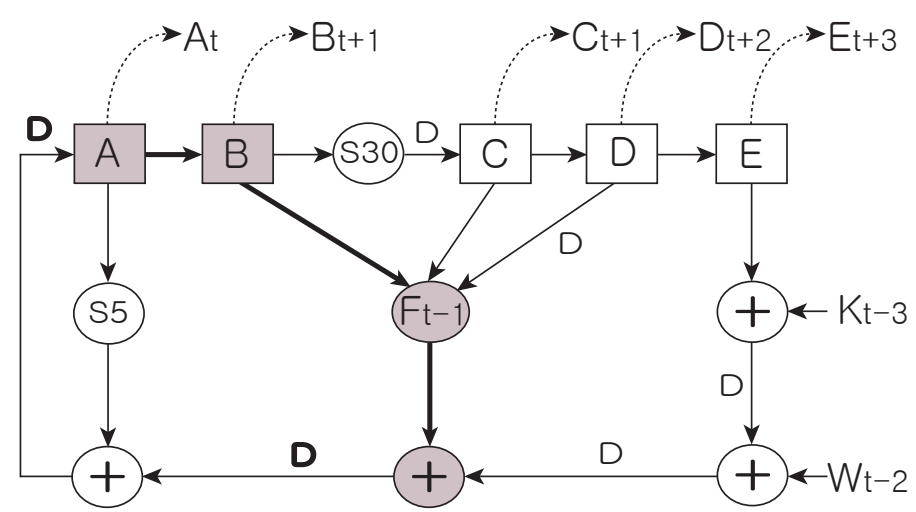

(a) DFG without CSA

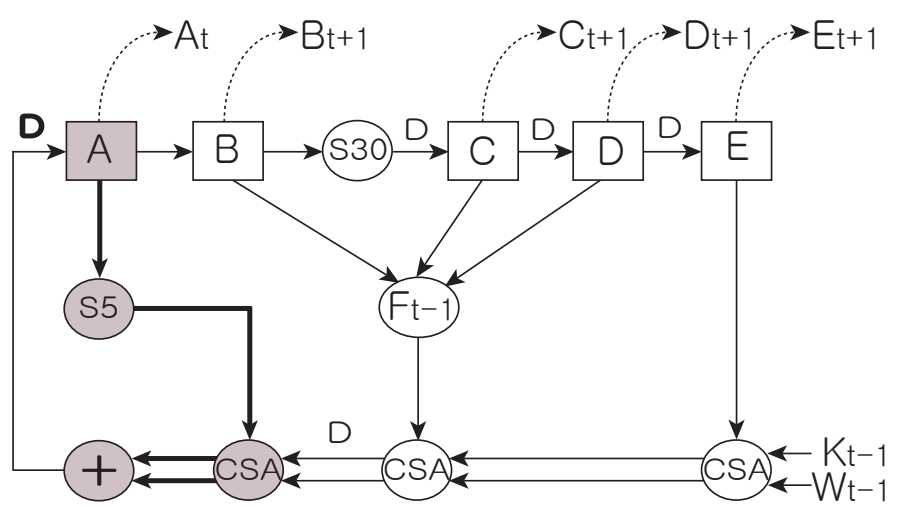

(b) DFG with CSA

Fig. 6. Retiming Transformation of SHA1

a ripple carry adder (which is very slow), the required gates are the same as CSA. However fast adders such as carry look-ahead adders will require a significantly larger number of logic gates. Therefore, the use of CSA rather than an adder in throughput optimum architecture will save gate area.

Due to the retiming transformation, some of the square nodes are no longer paired with algorithmic delays. Therefore, care must be used to properly initialize the registers and extract the final result: this is explained in Section 6 .

\subsection{Applying the Unfolding Transformation}

In order to achieve the iteration bound, the unfolding transformation is required. By expanding Eq. 8, we get Eq. 11. Since the register values of time index $t+2$ can be expressed using registers only with the time index $t$, the unfolded DFG with unfolding factor 2 can be derived using Eq. 11. Note that the 


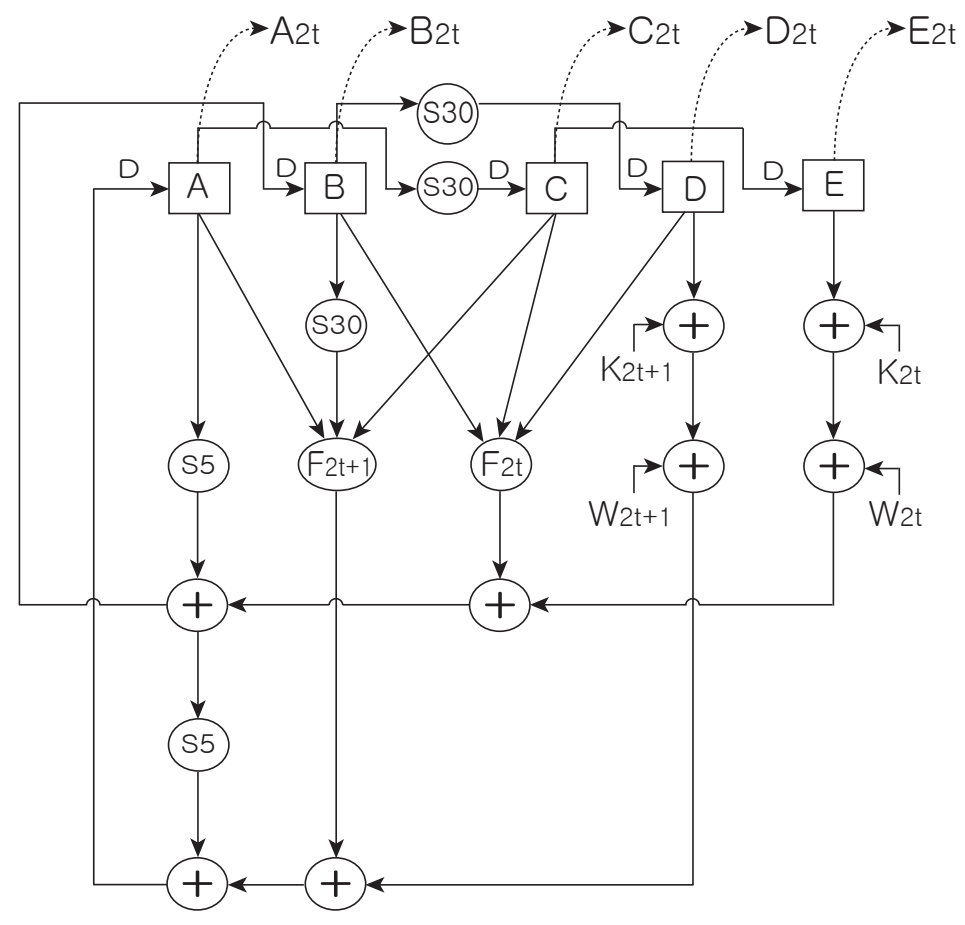

Fig. 7. Unfolding Transformation of SHA1

functional nodes are doubled due to the unfolding transformation.

$$
\begin{aligned}
& T E M P_{t}=S 5\left(A_{t}\right)+F_{t}\left(B_{t}, C_{t}, D_{t}\right)+E_{t}+W_{t}+K_{t} ; \\
& \begin{array}{l}
T E M P_{t+1}=S 5\left(A_{t+1}\right)+F_{t+1}\left(B_{t+1}, C_{t+1}, D_{t+1}\right)+E_{t+1}+W_{t+1}+K_{t+1} \\
\quad=S 5\left(T E M P_{t}\right)+F_{t+1}\left(A_{t}, S 30\left(B_{t}\right), C_{t}\right)+D_{t}+W_{t+1}+K_{t+1} ; \\
E_{t+2}=D_{t+1}=C_{t} ; \quad D_{t+2}=C_{t+1}=S 30\left(B_{t}\right) ; \quad C_{t+2}=S 30\left(B_{t+1}\right)=S 30\left(A_{t}\right) ; \\
B_{t+2}=A_{A+1}=T E M P_{t} ; \quad A_{t+2}=T E M P_{t+1} ;
\end{array}
\end{aligned}
$$


Since the iteration bound of (a) is smaller than (b) in Fig. 5, we perform the unfolding transformation on Fig. 5 (a), which results in Fig. 7. Usually the iteration bound can be achieved with the unfolding factor of the denominator of the iteration bound. In this case, note that in the indexes of $F, K$ and $W$, $t$ is replaced by $2 t$ due to the unfolding factor of two.

After the unfolding transformation, we can substitute two consecutive adders with one CSA and one adder. We have shown in the previous sub-section that using CSA does not reduce the iteration bound and therefore does not improve the throughput. However, since CSA occupies less area than a throughput optimized adder, we substitute adders with CSA whenever it is possible. Fig. 8 shows the



Fig. 8. Unfolding Transformation of SHA1 with CSA 
DFG which uses CSA. Some consecutive adders are not replaced by CSA since doing so will increase the iteration bound.

Since $3 \times \operatorname{Prop}(C S A)<\operatorname{Prop}(+)$, the loop having the maximum loop delay is the loop marked with bold lines in Fig. 8, and the normalized critical path delay, $\hat{T}$, is now equal to the iteration bound as shown in Eq. 12.

$$
\hat{T}=\frac{2 \times \operatorname{Prop}(+)+\operatorname{Prop}\left(F_{t}\right)}{2}=\operatorname{Prop}(+)+\frac{\operatorname{Prop}\left(F_{t}\right)}{2}=T_{\infty}^{(a)}
$$

Finally after performing the proper retiming transformations, we get the result of Fig. 9. The critical path is the path of shaded nodes in Fig. 9 (i.e. $S 5 \longrightarrow+\longrightarrow A \longrightarrow F_{2 t+1} \longrightarrow+$ ) and the critical path delay is $2 \times \operatorname{Prop}(+)+\operatorname{Prop}\left(F_{t}\right)$, which achieves the iteration bound. Note that there is no propagation delay on $S 5$ and $A$.

\section{Some Other MD4-based Hash Algorithms}

The proposed design methodology can be applied to any other MD4-based hash algorithm. In this section we apply the methodology to MD5 and RIPEMD-160.

\subsection{Iteration Bound and DFG Transformation of MD5}

MD5 [21] is developed by Ron Rivest in 1992 to improve MD4. MD5 is composed of 4 rounds, and each round is composed of 12 hash operations and produces a 128-bit output. The equation and the DFG of MD5 is shown in Eq. 13 and Fig. 10(a) respectively, where $S_{t}$ represents the shift function whose number of shift positions depends on the time index $t . X_{t}$ is a selection of expanded message words and $T_{t}$ is a constant whose value depends on $t$.

$$
\begin{aligned}
& T E M P_{t}=S_{t}\left\{A_{t}+F_{t}\left(B_{t}, C_{t}, D_{t}\right)+X_{t}+T_{t}\right\}+B_{t} ; \\
& D_{t+1}=C_{t} ; \quad C_{t+1}=B_{t} ; \quad B_{t+1}=T E M P_{t} ; \quad A_{t+1}=D_{t} ;
\end{aligned}
$$

Since the loop $\left(B \longrightarrow F_{t} \longrightarrow+\longrightarrow S_{t} \longrightarrow+\underset{D}{\longrightarrow} B\right)$ of the shaded nodes in Fig. 10(a) has the maximum loop bound, the iteration bound is shown in Eq. 14. Note that CSA cannot be used in this 




Fig. 9. Unfolding and Retiming Transformation of SHA1

loop since two adders are separated by a shift function. Though the use of CSA does not reduce the iteration bound, i.e. no benefit in throughput, we can use a CSA in $A_{t}+X_{t}+T_{t}$ to save some area.

$$
T_{\infty}=\max _{l \in L}\left\{\frac{t_{l}}{w_{l}}\right\}=2 \times \operatorname{Prop}(+)+\operatorname{Prop}\left(F_{t}\right)
$$

This iteration bound can be achieved by the retiming transformations shown on Fig. 10(b), where the critical path is marked by bold line $\left(B \longrightarrow F_{t} \longrightarrow+\longrightarrow S_{t} \longrightarrow+\right)$. The iteration bound for MD5 is double than that of SHA1. Thus when implemented in a similar technology, we expect that one hash operation of MD5 is half the speed of SHA1. 


\subsection{Iteration Bound and DFG Transformation of RIPEMD-160}

RIPEMD-160 [22] is designed by Hans Dobbertin et al. in 1996. RIPEMD-160 is composed of two parallel iterations, where each iteration is composed of 5 rounds, and each round is composed of 16 hash operations. The equation and DFG of RIPEMD-160 are shown in Eq. 15 and Fig. 11(a) respectively.

$$
\begin{aligned}
& T E M P_{t}=S_{t}\left\{A_{t}+F_{t}\left(B_{t}, C_{t}, D_{t}\right)+X_{t}+K_{t}\right\}+E_{t} ; \\
& E_{t+1}=D_{t} ; \quad D_{t+1}=S 10\left(C_{t}\right) ; \quad C_{t+1}=B_{t} ; \quad B_{t+1}=T E M P_{t} ; \quad A_{t+1}=E_{t} ; \\
& T E M P_{t}^{\prime}=S_{t}^{\prime}\left\{A_{t}^{\prime}+F_{t}^{\prime}\left(B_{t}^{\prime}, C_{t}^{\prime}, D_{t}^{\prime}\right)+X_{t}^{\prime}+K_{t}^{\prime}\right\}+E_{t}^{\prime} ; \\
& E_{t+1}^{\prime}=D_{t}^{\prime} ; \quad D_{t+1}^{\prime}=S 10\left(C_{t}^{\prime}\right) ; \quad C_{t+1}^{\prime}=B_{t}^{\prime} ; \quad B_{t+1}^{\prime}=T E M P_{t}^{\prime} ; \quad A_{t+1}^{\prime}=E_{t}^{\prime} ;
\end{aligned}
$$

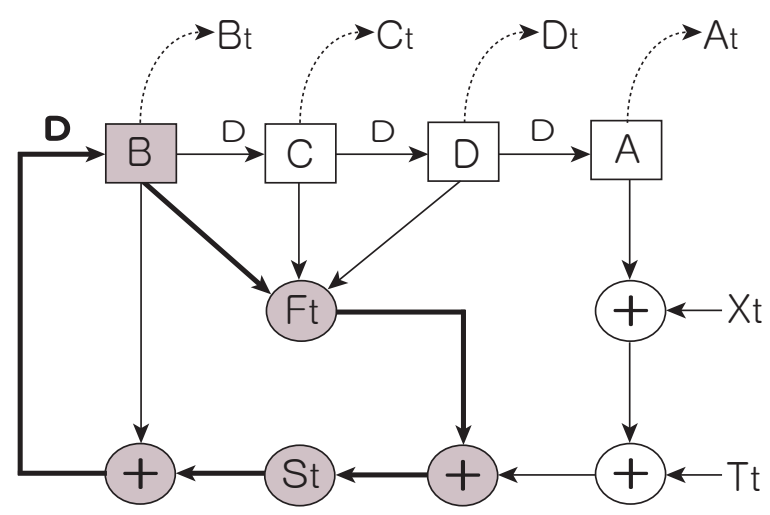

(a) Data Flow Graph

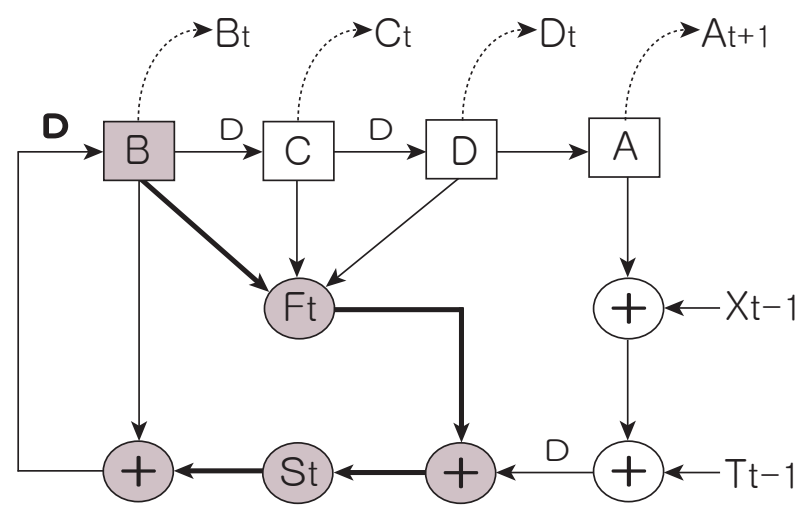

(b) Retiming Transformation

Fig. 10. MD5 Data Flow Graph and its transformation 


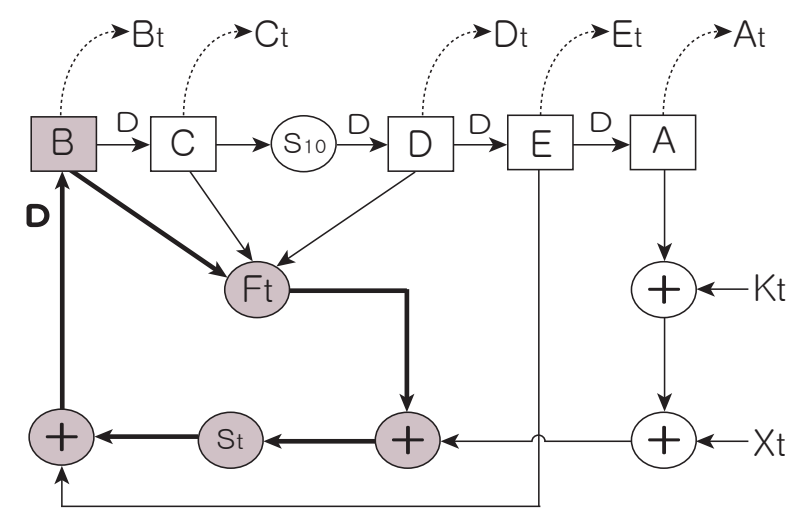

(a) Data Flow Graph

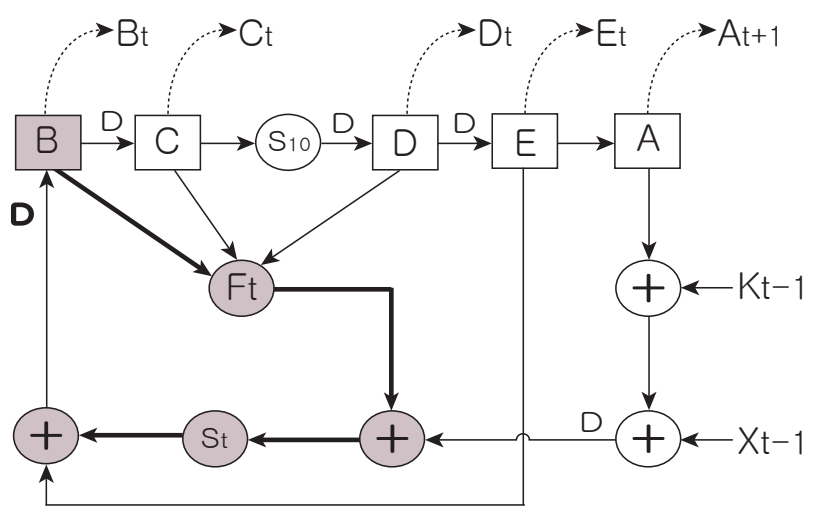

(b) Retiming Transformation

Fig. 11. RIPEMD-160 Data Flow Graph and its transformation

From Eq. 15 we can see that the two parallel iterations of RIPEMD-160 have identical DFG's. Therefore, we need to analyze only one part and then replicate the results for the second part. $S_{t}$ is a shift function, $X_{t}$ is a selection of expanded message words and $K_{t}$ is a constant whose value depends on the time index $t$.

The loop having the maximum loop bound, i.e. $B \longrightarrow F_{t} \longrightarrow+\longrightarrow S_{t} \longrightarrow+\longrightarrow{ }_{D} B$, is shown in Fig. 11(a) using shaded nodes and its iteration bound is shown in Eq. 16.

$$
T_{\infty}=\max _{l \in L}\left\{\frac{t_{l}}{w_{l}}\right\}=2 \times \operatorname{Prop}(+)+\operatorname{Prop}\left(F_{t}\right)
$$

The retiming transformation of RIPEMD-160 which achieves the iteration bound is shown in Fig. 11(b), where the critical path is marked by bold line $\left(B \longrightarrow F_{t} \longrightarrow+\longrightarrow S_{t} \longrightarrow+\right)$. The iteration bound of RIPEMD-160 is the same as for MD5 and it is double that of SHA1. 


\section{Synthesis of the SHA1 Algorithm}

In order to verify our design methodology, we synthesized SHA1 for an ASIC using a TSMC 0.18 $\mu m$ CMOS standard cell library. We verified that the actual critical path occurs as predicted by our analysis and that the hash outputs are correct. We synthesized two versions: one after only the retiming transformation and the other after both the unfolding and retiming transformations. Since the unfolding transformation introduces duplications of functional nodes, its use often incurs a significant increase in area. For the version using only the retiming transformation, we select the DFG of Fig. 6(b). Though they have the same iteration bound, Fig. 6(b) has less area than Fig. 6(a) due to the use of CSA. Another benefit of Fig. 6(b) is a smaller number of overhead cycles than Fig. 6(a), which will be explained in this section. For the version of the unfolding and retiming transformation, we synthesized the DFG of Fig. 9.

\subsection{SHA1 with the Retiming Transformation}

In the transformed DFG's, some of the register values, i.e. $A, B, \ldots, E$, are no longer paired with an algorithmic delay $D$. This means that the register $B$ is no longer necessary except for providing the initial value. The retiming transformation moves the delay $D$ associated with register $B$ between two CSA adders (we name this delay $\mathrm{T}$ ). Though the size of $\mathrm{T}$ is doubled (to store both the sum and carry values produced by the CSA), our experiments showed a smaller gate area in Fig. 6(b) than Fig. 6(a) due to the small size of CSA.

Another difference occurs during initialization. In the original DFG (Fig. 5(b)), all the registers are initialized in the first cycle according to the SHA1 algorithm. In contrast, initialization requires two cycles in the retimed DFG (Fig. 6(b)). This is because there should be one more cycle to propagate initial values of $B, C, D$ and $E$ into $T$ before the DFG flow starts. The procedure to update the registers is shown in Fig. 12 where all the sub-scripts are ignored for simplicity.

In the first cycle, the values of $A, B, C, D$ and $E$ are initialized as the SHA1 algorithm. At the second cycle, $A$ holds its initial value and $C, D, E$ and $T$ are updated using the previous values of $B$, $C, D$ and $E$. From the third cycle, the registers are updated according to the DFG (Fig. 6(b)). Note that the register $B$ does not overlap with the register $T$ in their use and hence $T$ can be reused to hold $B_{\text {init }}$ at the first cycle. 




Fig. 12. Register Update Procedure of retimed SHA1

Due to two cycles of initialization, the retimed DFG introduces one overhead cycle. This fact can also be observed noting that there are two algorithmic delays from $E$ to $A$. In order to update $A$ with a valid value at the beginning of the iteration, two cycles are required for the propagation. In the case of the retimed SHA1 without CSA (Fig. 6(a)), there are three overhead cycles due to the four algorithmic delays in the path from $E$ to $A$.

Therefore, the required number of cycles for Fig. 6(b) is the number of iterations plus two cycles for initialization, which results in 82 cycles. Since the finalization of SHA1 can be overlapped with the initialization of the next message block, one cycle is excluded from the total number of cycles.

When extracting the final results at the end of the iterations, we should note the indexes of registers. In Fig. 6(b), the index of the output extraction of the register $A$, i.e. $A_{t}$, is one less than the others. Therefore, the final result of the register $A$ is available one cycle later than the others.

\subsection{SHA1 with the Unfolding and Retiming Transformation}

In the case of Fig. 9, there are 6 algorithmic delays and two of them are not paired with a square node. We name the register for the algorithmic delay between two adders $T 1$ and the register for the algorithmic delay between an adder and $S 5 T 2$. However, since $T 2$ is equivalent to $B$, we do not need a separate register for $T 2$. Therefore, the total required registers remain at 5 (retiming does not introduce extra registers in this case). The register updates of this case are described in Fig. 13.

Note that there is no overlapped use of $A$ and $T 1$ and hence $T 1$ can be used to hold $A_{\text {init }}$ at the first cycle. Since there is only one algorithmic delay in all the paths between any two consecutive square nodes, there is no cycle overhead resulting in the total number of cycles of 41, i.e. 40 cycles for iterations plus one cycle for initialization. When extracting the final result of $A$, the value must be driven from $+(S 5(B), T 1)$. This calculation can be combined with the finalization since the combined 


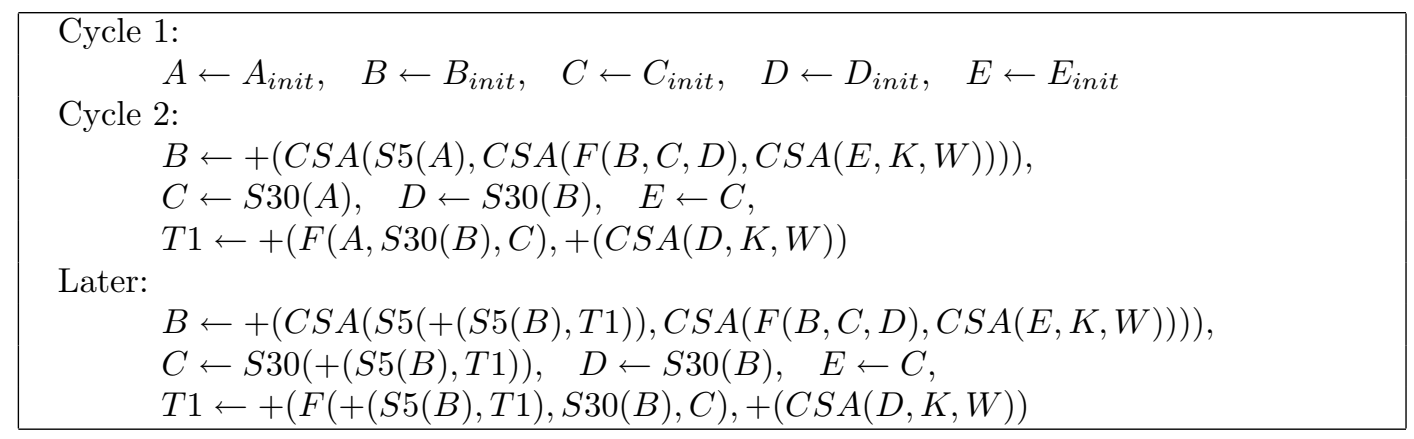

Fig. 13. Register Update Procedure of retimed and unfolded SHA1

computational delay of $+(S 5(B), T 1)$, whose delay is $\operatorname{Prop}(+)$, and the finalization, whose delay is $\operatorname{Prop}(+)$, is $2 \times \operatorname{Prop}(+)$ which is less than the critical path delay.

\subsection{Synthesis Results and Comparison}

The synthesis results are compared with some previously reported results in Table 1 . The 82 cycle version is made by the retiming transformation (Fig. 6(b)), and the 41 cycle version is made by the retiming and the unfolding transformations together (Fig. 9). The throughputs are calculated using the following Eq. 17.

$$
\text { Throughput }=\frac{\text { Frequency }}{\# \text { of Cycles }} \times(512 \text { bits })
$$

In Table 1, the work of [12] is a unified solution for MD5, SHA1 and RIPEMD160 so that the gate count is quite large. [13] used $0.13 \mu m$ technology but it is much slower than our proposal which used

Table 1. The comparison between our results and others

\begin{tabular}{|c|c|c|c|c|c|}
\hline & $\begin{array}{c}\text { Technology } \\
\text { (ASIC) }\end{array}$ & $\begin{array}{c}\text { Area } \\
\text { (Gates) }\end{array}$ & $\begin{array}{c}\text { Frequency } \\
\text { (MHz) }\end{array}$ & Cycles & $\begin{array}{c}\text { Throughput } \\
\text { (Mbps) }\end{array}$ \\
\hline$[11]$ & $0.25 \mu$ & 20,536 & 143 & 82 & 893 \\
\hline$[12]^{*}$ & $0.18 \mu$ & 70,170 & 116 & 80 & 824.9 \\
\hline$[13]$ & $0.13 \mu$ & 9,859 & 333.3 & 85 & 2,006 \\
\hline$[17]$ & $0.18 \mu$ & 54,133 & 72.7 & 12 & 3,103 \\
\hline$[19]$ & $0.18 \mu$ & 23,000 & 290 & 82 & 1,810 \\
\hline Our Proposal & $0.18 \mu$ & 19,767 & 584.8 & 82 & 3,651 \\
& & 23,167 & 299.4 & 41 & 3,738 \\
\hline
\end{tabular}

*This is a unified solution for MD5, SHA1 and RIPEMD-160. 
$0.18 \mu m$ technology. The architecture of [17] has a small cycle number and a large gate area due to the unfolding transformation with a large unfolding factor of 8. Even with the use of a large unfolding factor, its throughput is still smaller than our proposal. Comparing our 41 cycle version with [19], the areas and the frequencies are similar but our version has only half the cycles resulting in twice the throughput.

Though the 41 cycle version of our proposals achieves the iteration bound with throughput optimum architecture, the 82 cycle version can be a good tradeoff since its gate area is much smaller with slightly less throughput.

Our designs were described at the register transfer level and we have concentrated on optimizing at micro-architecture level rather than focusing on lower-level optimizations. In other words, we let standard synthesis tools generate our adders in standard CMOS logic and use generic architectures. While higher throughput can be achieved with faster adder architecture and/or faster logic families, we show that iteration bound analysis still determines the optimum high level architecture of an algorithm. We validate these claims by producing designs that have the highest throughputs among all published results.

\section{Conclusion}

We propose a design methodology for throughput optimum architecture of MD4-based hash algorithms. This methodology is very strong since the upper limit for the maximum throughput can be obtained by a mathematical method and the optimum architecture can be systematically designed. Though straightforward, the transformations sometimes produce architectures which necessitate careful implementation. These issues are discussed and design examples using some popular hash algorithms such as SHA1, MD5 and RIPEMD-160 are used to illustrate how they are resolved.

In order to verify the design methodology we synthesized the SHA1 algorithm, which is the algorithm requiring all the procedure we preset among our examples. According to our synthesis results in a $0.18 \mu \mathrm{m}$ CMOS technology, the maximum achievable throughput is 3,738 Mbps, which is much faster than previously reported implementations. The optimized architecture for SHA1 that we have developed conclusively shows the effectiveness of our design methodology.

Our proposed design methodology can also be used by hash algorithm designers who wish to use high throughput as a design criteria. 
Acknowledgments. This work is supported by NSF CCF-0541472, FWO and funds from Katholieke Universiteit Leuven.

\section{References}

1. Digital Signature Standard. National Institute of Standards and Technology. Federal Information Processing Standards Publication 186-2. http://csrc.nist.gov/publications/fips/fips186-2/fips186-2-change1.pdf.

2. K.K. Parhi: VLSI Digital Signal Processing Systems: Design and Implementation. Weley. Pp. 43-61 and pp. 119-140. 1999.

3. A. Menezes, P. van Oorschot and S. Vanstone: Handbook of Applied Cryptography. CRC Press. 1996. Section 9.4.2. P. 343 .

4. L. Dadda, M. Macchetti and J. Owen: An ASIC design for a high speed implementation of the hash function SHA-256 (384, 512). ACM Great Lakes Symposium on VLSI. Pp. 421-425. 2004.

5. L. Dadda, M. Macchetti and J. Owen: The design of a high speed ASIC unit for the hash function SHA-256 (384, 512). Proceedings of the conference on Design, Automation and Test in Europe (DATE'04). IEEE Computer Society. Pp. 70-75. 2004.

6. M. Macchetti and L. Dadda: Quasi-pipelined hash circuits. Proceedings of the 17th IEEE Symposium on Computer Arithmetic (ARITH'05). Pp. 222-229. 2005.

7. H. Michail, A.P. Kakarountas, O. Koufopavlou and C.E. Goutis: A Low-Power and High-Throughput Implementation of the SHA-1 Hash Function. IEEE International Symposium on Circuits and Systems (ISCAS'05). Pp. 4086-4089. 2005.

8. R. P. McEvoy, F. M. Crowe, C. C. Murphy and W. P. Marnane: Optimisation of the SHA-2 Family of Hah Functions on FPGAs. Proceedings of the 2006 Emerging VLAI Technologies and Architectures (ISVLSI'06). Pp. 317-322. 2006.

9. F. Crowe, A. Daly and W. Marnane: Single-chip FPGA implementation of a cryptographic co-processor. Proceedings of the International Conference on Field Programmable Technology (FPT'04). Pp. 279-285. 2004.

10. R. Lien, T. Grembowski and K. Gaj: A 1 Gbit/s partially unrolled architecture of hash functions SHA-1 and SHA-512. CT-RSA 2004. Vol. 2964 of LNCS. Springer. Pp. 324-338. 2004.

11. Y. Ming-yan, Z. Tong, W. Jin-xiang and Y. Yi-zheng: An Efficient ASIC Implementation of SHA-1 Engine for TPM. The 2004 IEEE Asia-Pacific Conference on Circuits and Systems. Pp. 873-876. 2004.

12. T. S. Ganesh and T. S. B. Sudarshan: ASIC Implementation of a Unified Hardware Architecture for NonKey Based Cryptographic Hash Primitives. Proceedings of the International Conference on Information Technology: Coding and Computing (ITCC'05). Pp. 580-585. 2005. 
13. A. Satoh and T. Inoue: ASIC-Hardware-Focused Comparison for Hash Functions MD5, RIPEMD-160, and SHS. Proceedings of the International Conference on Information Technology: Coding and Computing (ITCC'05). Pp. 532-537. 2005.

14. M. Wang, C. Su, C. Huang and C. Wu: An HMAC Processor with Integrated SHA-1 and MD5 Algorihtms. Proceedings of the 2004 Asia and South Pacific Design Automation Conference (ASP-DAC'04). Pp. 456-458. 2004.

15. C. Ng, T. Ng and K. Yip: A Unified Architecture of MD5 and RIPEMD-160 Hash Algorithms. Proceedings of the 2004 International Symposium on Circuits and Systems (ISCAS'04). Pp. 889-892. 2004.

16. K. Järvinen, M. Tommiska and J. Skyttä: Hardware Implementation Analysis of the MD5 Hash Algorihtm. Proceedings of the 38th Annual Hawaii International Conference on System Science (HICSS 05). P. 298. 2005 .

17. Y. K. Lee, H. Chan and I. Verbauwhede: Throughput Optimized SHA-1 Architecture Using Unfolding Transformation. IEEE 17th International Conference on Application-specific Systems, Architectures and Processors (ASAP'06). Pp. 354-359. 2006.

18. Y. K. Lee, H. Chan, and I. Verbauwhede: Iteration Bound Analysis and Throughput Optimum Architecture of SHA-256 (384,512) for Hardware Implementations. Information Security Applications, 8th International Workshop, WISA 2007, Lecture Notes in Computer Science 4867, S. Kim, H. Lee, and M. Yung (eds.), Springer-Verlag, pp. 102-114, 2007.

19. Helion SHA-1 hashing cores. Helion Technology. http://heliontech.com/sha1.htm.

20. Secure Hash Standard. National Institute of Standards and Technology. Federal Information Processing Standards Publication 180-2, http://csrc.nist.gov/publications/fips/fips180-2/fips180-2.pdf

21. R. Rivest: The MD5 Message-Digest Algorithm. http://www.faqs.org/rfcs/rfc1321.html.

22. H. Dobbertin, A. Bosselaers and B. Preneel: RIPEMD-160: A Strengthened Version of RIPEMD. http://homes.esat.kuleuven.be/ ${ }^{\sim}$ cosicart/pdf/AB-9601/AB-9601.pdf. 